\title{
Tratamiento familiar sistémico de los niños con problemas conductuales o emocionales
}

\author{
Hernán Montenegro A. ${ }^{1}$
}

\begin{abstract}
Resumen
Se expone- los conceptos más relevanles de la teoria general de sislemas y de la cibernética, que fundamentan el entcque sistemico familiar pera Irarar problemas conductuales y errocionales de la niñez y juvenlud, la forma en que esle opera en la piáctica clínica y sus diferencias con otras orieniaciones teóricas, en particula: el cambio del oco de atention desde el individuo $c$ sus procesos iritrapsicuicos a su interacción cemo parte de un sistema mayor y su cambio de mirada desce los contenidos del probema a los procesos estructurales y contextos de las concivcras. Se lustra el entoque con ocs historias clínicas de riños tratados con él.
\end{abstract}

Palabras clave:trasionos condustuales, trostornos emotionales, niños, tratamiento farpiliar sistémico.|

\section{Systemic family therapy for children with and behavioral emotional problems}

The moin corceprs under the general theory of systems and cibermetics ore described os they provide founations to the systerric fornily aproach to theraphy of emotional and behavioral conflicts in chilaren. Focus changes from irdividuals and intrapsyquic processes to persendi iriterations as $c$ parl of the family as a system, and from problem contents to structurol processes and situations are emphosized The clinical cases of wo children who were treated with this aporoach a'e cescribed to illustrale how hey work.

'Key words: behov oro', emotional, disorders, systemic family therapy.'

No obstante la amplitud del tema de esta exposición y las limitaciones de espacio disponible se revisará sucintamente el marco teórico que sustenta el enfoque de la terapia familiar sistémica y se expondrán dos ejemplos de cómo este opera en la práctica clínica.

Los términos terapia familiar y terapia familiar sistémica (TFS) no son sinónimos ni equivalentes. Existen terapeutas de orientación psicodinámica que hacen terapia familiar, como así mismo de orientación cognitiva conductual que reúnen a todos los miembros de una familia en un tratamiento. Lo que diferencia a la terapia familiar sistémica es su fundamento en la teoría general de los sistemas y la cibernética. Bajo ese gran alero teorico se han desarrollado varios "modelos intermedios" que, en general, se diferencian unos de otros más que nada en el énfasis que ponen en los distintos conceptos de la teoría (totalidad, límites, jerarquía, homeostosis, retroalimentación, entropía, procesos estructurales, etc). Así, por ejemplo, existen el modelo estructural de S. Minuchin, el modelo estratégico de Palo Alto, el modelo de Milán derivado del anterior, el constructivista de Von Foester, Maturana y Varela, entre otros. Lo que los distingue no es el número de miembros de la familia que se citan a las sesiones o si estas integran a otros parientes o miembros de la red social de una familia sino el enfoque sistémico cibernético. Así entonces podrán haber terapias familiares sistémicas, terapia de pareja sistémicas y terapia individual sistémica'.

I. Deparıamento Psiquiarría Sur, Facultad de Medicina. Universidad de Chile. Director Instituto de Terapia Familiar de Santiago. 


\section{Conceptos básicos}

La tcoría general de sistemas ${ }^{2}$ representa una nueva epistemología generada como reacción o propuesta altemaliva al pensamiento analíticoreduccionista y mecanicista que informaba la forma de conocer empleada por la ciencia hasta entonces. $S_{u}$ insatisfacción deriv 6 de las limitaciones que tenía la lorma de explicar los fenómenos o eventos mediante series lineales de causas y efectos en cadena, cada uno develando un evento causal cono antecedente del siguiente. Como alternativa propone examinar la estructura lógica de los sistemas. Postula que la organización, más que la reducción, es el principio unificador básico de las ciencias. De acuerdo a esta teoria, se define el concepto de sistemas como el conjunto de elementos (partes o eventos) relacionados entre sí y que eștán en constante interacción, de una manera específica (consistente o predecible), formando una unidad o totalidad.

Los sistemas pueden ser abiertos o cerrados. Los biológicos son sjempre abiertos, lo mismo que los sociales e intercambian materia, energía e información con otros sistemas.

La fanilia es un sistema abjerto, compuesto $p(r$ subsistemas (parentil, conyugal, fraterno), que -a su vez-contienen otros subsistemas individuales en interacción no sólo entre sí, sino también con otros sistemas sociales. Desde esta óptica los fenómenos son entendidos en su totalidad, tomando en cuenta el todo que los comprende y del que forman parte a través de su interacción, de los patrones relacionales y del contexto en que ocurren.

Esta teoria hace un llamado a la necesidad de un enfoque globalizador e interdiciplinario de los fenómenos. Su interés es considerar la interrelaci6n y dependencia mulua de las partes de un todo u objeto que tenga como propósito un conjunto de metas determinadas.

Un sistema siempre está compuesto de un conjunto de partes o elementos que están en una relación dada consistente u organización. En toda organización se dan ciertas características básicas: totalidad, límiles, jerarquía, control, homeoslasis, entropía ticmpo y espacio.

De la totalidad deriva una de las leyes de oro del enfoque sistémico, la que dice que "el todo no es igual a la suma de sus partes", pues el todo confiere a sus partes características que no poseen individualmente y es la interacción entre las partes la que genera o produce una totalidad.

Los límites existen en todos los sistemas y son la regiones que separan un sistema de otro y que se sitúan en una gradiente de mayor o menor permeabilidad de intercambios con su medio.

Los sistemas están organizados en forma jerárquica. No todas las partes de un sistema funcionan a un mismo nivel, pudiendo, por ejemplo, funcionar como partes de un sistema mayor.

El concepto de control tiene que ver con los factores que mantienen el equilibrio y estabilidad del sisterna. Opera mediante la retroalimentación, que es el proceso por el cual el sistema regula sus respuestas (salida) a los estímulos externos (entradas).

La homeostasis es el estado constante o estabilidad de un sistema que, en general, se mantiene mediante mecanismos de retroalimentación negativa.

La entropía del sistema es un concepto derivado de la termodinámica. Se refiere a la degradación natural de la energía a lo largo del tiempo, que lleva a los sistemas del orden al desorden, a la desintegración o la muerte. En los sistemas vivos opera una entropía negativa, que los lleva a una estructuración mayor a lo largo del tiempo. En este sentido el rol que juega la información es esencial.

Todos los sistemas operan dentro del tiempo y el espacio. Si se enfoca la atención en la forma como las partes se organizan en el espacio se definirán sus estructuras; si la variable temporal es la que nos preocupa se hablará de sus procesos. Hay una interacción entre ambas dimensiones. siendo de hecho inseparables.

La cibernética en una teoría más bien reciente que se desarrolló en forma más o menos paralela con la teoría general de los sistemas, con la que comparte muchos elementos ${ }^{3}$. Investigadores de diversas disciplinas se interesaron por conocer la organización de distintos eventos. Piaget se preocupaba de cómo los niños organizan los procesos del conocer; Gregory Bateson. antropólogo, estudiaba, en Nueva Guinea, cómo las conductas rituales se organizan en la interacción social. Se comenzó a reconocer que la organización de los eventos, sean neurológicos, psicológicos, conductuales o sociales, podía comprenderse en términos de información más 
que en términos de encrgía y materia. Fue Wiener, un matemático, quien propuso el término cibernética para esta nueva forma de pensamiento. La cibernética sería la ciencia de la información, de los patrones, de las formas y de la organización, que no tienen que ver con lo material. Es como un mundo invisible, por ejemplo, en el campo de los sistemas faniliares, la secuencia de acción en un episodio. De allí surge la necesidad de reemplazar nuestra común forma de pensar lineal-causal ( $A$ es causa de $B$ o esta es efecto de A) al pensamiento circular cibernético. De esta manera, podemos decir que en la interacción de un sistema compuesto de 4 miembros, A afecta a B, este a C, quien afecta a $D$, el que afecta a $A$ y así sucesivamente hasta el infınito. De esta forma, cualquiera puntualización de la realidad que se haga, en el sentido de decir que la conducta de cualesquiera de ellos es la "causa" de la conducta de otro, no es sostenible. La idea central de la tcoría cibernética es la de retroalimentación, que Wiener defïne como el método de control de un sistema que ocurre al reinsertar en él los resultados de su comportamiento, actuación o conducta pasada. El ejemplo clásico es la regulación de la calefacción por un termostato. En una fanilia este puede ilustrarse por el comienzo de una discusión entre dos de sus miembros, que genera una escalada (como la temperatura de una casa) hasta que sc alcanza un umbral intolerable para el sistema. Ese umbral es, a veces, regulado o definido -como un termostato- por otro miembro, quien desarrolla una determinada conducta que detiene la discusión. Todas las familias tienen procesos de retroalimentación que proveen estabilidad para la organización de toda ella. Los procesos cibernéticos nunca son estáticos. Pueden darse amplificaciones de las desviaciones del objetivo. En la medida que esta amplificación prođucida por un proceso de retroalimentación positiva no es regulada por otra retroalimentación mayor, el sistema se destruye.

\section{Terapia familiar sistémica en la práctica clínica}

Por lo general en la terapia familiar sistémica deben ser incluidos todos los miembros directos de la familia en la evaluación inicial, a menos que motivos insubsanables lo impidan. Lo mis- mo deberá ocurrir en las sesiones siguientes, luego de establecerse el contrato terapéutico. Las sesiones se fijan semanal o quincenalmente. Los niños pequeños, contrariamente a lo que podría suponerse, pueden constituir valiosas fuentes de información por medio de sus expresiones o conductas. Puede ocurrir que los miembros de la familia extendida también sean relevantes. En el caso de padres separados, se trabaja en sesiones distintas con los hijos y cada uno de los padres. El foco de atención está puesto en el aquí y ahora, no en el pasado. Se trata de producir cambios en los patrones de interacción y no de obtener percepciones interiores. Interesan las interacciones del sistema para revelar las funciones que cumple el sintoma del paciente índice.

Otro elemento central en la terapia sistémica familiar, que tiene que ver con sus orígenes, es la comunicación. De hecho, varios pioneros en este campo y en la teoría de la comunicación, como Don Jackson. Jay Haley, Virginia Satir, Gregory Bateson y Paul Watzlawick, comenzaron a interesarse en este nuevo enfoque al estudiar las familias de pacientes esquizofrénicos. El sintoma se considera como una solución protectora. El portador se sacrifica a sí mismo para defender la homeostasis del sistema. El terapeuta familiar ve a la familia como un organismo y a esta protección, no como a propósito o como una respuesta ayudadora, sino como una reacción del organismo bajo el estrés. Los otros miembros de la familia son considerados igualmente sintomáticos. Se trata de desafiar la definición que hace la familia del problema y la forma en que ellos responden. Asf, pues, es un cambio en el foco de atención, ya que no es el individuo o sus procesos intrapsíquicos, sino su interacción como parte de un sistema mayor lo que interesa. Se cambia la mirada desde los contenidos del problema a los procesos estructurales y contextos en que la conducta ocurre; desde la motivación de su conducta a los efectos que esta produce (interesa más el para qué, que el porque de sus conductas). Importa, por lo tanto, averiguar qué le dice el síntoma a los demás. qué les hace hacer a los demás y qué modificación en el sistema de roles produce o qué beneficio le reporta a cada miembro del sistema. Puede resultar igualmente de interés elucidar qué conflictos oculta o cómo contribuye cada miembro de la familia a perpetuarlos. 
Si bien es cierto que la psiquiatría infantil, desde sus inicios, ha incluido a los padres y a la familia en el diagnóstico y tratamiento de los diversos motivos de consulta, la diferencia con el abordaje sistémico familiar radica en el foco central. En las orientaciones tradicionales el foco central del diagnóstico está en el niño a quien se considera enfermo y la familia pasa a ser sólo el telón de fondo. En el enfoque familiar sistémico, en cambio, centra su atención en el sistema familiar, que así pasa a ser el paciente, junto con su interacción, quedando el sujeto índice o identificado por ella, en el trasfondo. Este pasa a ser un síntoma más de un sistema cuya disfunción es generadora del sufrimiento. Otro elemento distintivo lo constituye el rol del terapeuta quien, en el enfoque sistémico familiar, pasa a formar parte de un nuevo sistema (el sistema terapéutico) que gobierna la conducta de sus miembros.

Lo que hace que un sistema familiar consulte es el sufrimiento sentido a distinguido por sus propios miembros. Por lo tanto, desde esta perspectiva, es difícil para un observador externo establecer o fijar reylas estándares para considerar lo que es funcional o disfuncional en una familia, aun cuando se trate de un profesional de la saluo mental. Esta consideración es particularmente relevante cuando se trabaja con familias de bajo nivel económico o de grupos étnicos o religiosos distintos de la mayoría social. No obstante lo anterior, existe cierto consenso en cierlos indicadores de salud mental familiar.

En primer término el hecho que los límites generacionales estén celimitados de tal manera que la estructura jerárquica sea clara y definida, lo que significa que las figuras parentales son padres y no hermanos, ni hijos de sus hijos. Otro indicador es que los padres han logrado estar bien diferenciados o han desarrollado una identidad bien definida, antes de scpararse de sus respectivas familias de origen; de esta forma logran manicner una lealtad mayor con su familia actual que con la de procedencia. También es indicio positivo que los esposos se sitúan uno al otro ances que nadie más, incluidos por cierto los hijos (primero se es pareja y después se es padre). Se estimula el desarrollo de la identidad y autonomía de cada uno de los miembros de la familia. Existe espacio para el crecimiento de lodos y se fomenta la independencia gradual de los hijos, tolerando sus diferencias. Los hijos no son utilizados para resolver los problemas conyugales, ni solicitados para hacer alianzas con alguno de sus progenitores. Se expresan constantemente el afecto y la calidez, sin llegar a constituir sentimientos posesivos entre los padres y entre estos y los hijos. Idealmente, cultivando el sentido del humor. Existe una comunicación abierta, franca, directa y explícita, para tratar cualquier asunto, distinguiendo entre sentimientos y conductas. No existe miedo al conflicto, ya que este es inherente a las relaciones interpersonales: lo importante no es evitarlo, sino tratar de resolverlo. Existe la posibilidad de mantener relaciones abiertas con personas extrañas a la familia, como parientes, amigos, compañeros. Existe una capacidad de adaptarse a los cambios que son inherentes al ciclo de vida familiar y a los que las contingencias del vivir deparan.

A la inversa, se estima como indicadores de alteraciones de la salud mental familiar la comunicación confusa, evasiva, contradictoria y doble vinculada. Una actitud de desconfianza entre sus miembros. Incapacidad para adaptarse a cambios y, o, elaborar perdidas. Límites difusos entre los subsistemas. Tendencia a fijar roles, como por ejemplo chivos expiatorios. Alteración del sentido de realidad. Estructura caótica del poder o existencia de coaliciones de algunos miembros en contra de otro.

\section{Historias clínicas}

1. Un niño de 7 años, que llamaremos Miguel, fue traído a consulta por su madre debido a su extremada timidez, inseguridad, temor a estar solo, situación que se exacerba durante la noche, necesitando dormir con su madre. El niño no tiene amigos en el colegio o en su vecindario, donde hay otros de su edad. Su rendimiento escolar es más que satisfactorio. La familia está compuesta, además, por una hermana de cuatro años y el padre, empleado bancario. La madre es dueña de casa. En las sesiones diagnósticas quedó de manifiesto una dinámica familiar caracterizada por una relación matrimonial distante, donde existía una crítica permanente del padre hacia la esposa por la sobreprotección que esta brinda al niño. Ella, a su yez, reprocha al padre su falta de interés por interactuar con su 
hijo, en contraste con la atención que brinda a su hija. El padre, además, está constantemente reprendiendo a Miguel por su "falta de hombría" y "comportamiento de guagua". Esta dinámica interaccional se grafić además por el lugar que ocuparon físicamente durante estas primeras sesiones: el niño, teniendo la posibilidad de vcupar un asiento propio, se mantuvo de pie junto a la silla de la mamá, con la intención de ocupar lambién el asiento de ella. Cada vez que se le dirigía la palabra, miraba primero a su madre como pidiendo que respondiera por él. La niña por su parte se entrctuvo manipulando algunos juguetes que había en la sala e interactuando preferentemente con su padre.

Siguiendo el modelo estructural de Minuchin, en este caso se procedió primero a identiticar el problema estructural disfuncional. las pautas transaccionales del sistema y la circularidad de las interacciones: la madre sobreprotege al hijo para compensar el rechazo afectivo hacia él y su distanciamiento afectivo con ella por parte del padre. El padre por su parte exacerba su crítica a la conducta de su hijo para, desde su perspectiva, compensar o atenuar el riesgo de tener un hijo afeminado. Esta actitud también está movilizada por su percepción de ver en su hijo el receptor de un cariño materno que él no tecibe de su esposa. El niño, por su parte, mientras mấs rechazado es por su padre, más se refugia en su madre, con lo que de paso la gratifica efectivamente y esta se siente a su vez compensada de la lejanía afectiva de su cónyugue. Como puede apreciarse en esta circularidad, cualquiera puntuación que se hiciera en el sentido de identificar qué es causa, qué es consecuencia, es irrelevante.

Asi entonces y en contraste con lo que habría sido el abordaje terapéutico desde otros marcos téricos, se procedió a manejar el espacio físico de las sesiones separando y acercando a los distintos miembros de la familia. De esta manera se inició el proceso de cambiar las estructuras existentes, crear estructuras nuevas y bloquear las disfuncionales. Por ejemplo, en una sesión sc pidió a la mamá encargarse de entretener a la hija e interactuar sólo con ella utilizando el material de juego disponible. Entretanto el terapeuta facilitaba el diálogo y la interacción del padre con su hijo, inquiriendo de paso qué actividades les gustaría compartir a cada uno de ellos en su ticmpo libre.
Luego se procedió a fijar tareas que crearan nuevas maneras de interacción tendientes a romper la alianza madre hijo y fortalecer el vínculo de este con su padre. Un ejemplo fue que, al volver de su trabajo, el papá tomara la iniciativa de interiorizarse de las actividades escolares de Miguel o realizaran algún juego de mesa y que, paralelamente, los sábados en la mañana salieran los dos solos como rutina y por un tiempo, a andar en bicicleta o algún parque de entretenciones. En una sesión se citó a los padres solamente con el objeto de hacer explícita la circularidad de sus interacciones y redifinir el motivo de un sufrimiento, excluyendo a $\mathrm{Mi}$ guel de su condición de paciente identificado. Junto a esto se concitaron para fortalecer la relación y el espacio de pareja.

2. Oscar es un niño de 12 años, que inició un tratamiento por dificultades escolares a los 7 , cuando cursaba el segundo año básico. En esa época se diagnosticó síndrome del déficit atercional con hiperactividad y dislexia secundaria. Se indicó rehabilitación psicopedagógica y se prescribió paralelamente metilfenidato. El tratamiento se mantuvo hasta aproximadamente los 10 años en que se superó su trastorno especílico del aprendizaje y disminuyeron considerablemente los síntomas derivados del déficit atencional, manteniéndose hasta la fecha el medicamento, con lo cual su conducta en el colegio se mantiene dentro de límites normales. Oscar es miembro de una familia compuesta por ambos padres (45 años el padre y 40 años la madre) y tres hermanos más, Sara de 15 , Rubén de 10 y Carlos de 8 años. El motivo de consulta fue un conjunto de trastornos de la conducta de Oscar desde más o menos un año atrás, caracterizados por extrema violencia en el hogar, en especial con su madre y sus hermanos menores, desobediencia marcada e irritabilidad permanente. La vida matrimonial de los padres se vio interrumpida hace tres años por una separación transitoria que duró aproximadamente 6 meses. luego de lo cual la relación de pareja ha sido conflictiva.

En las sesiones diagnósticas se detect 6 alto grado de tensión entre los miembros de la familia, existiendo una clara alianza del paciente índice con su padre. Además se observó una importante discrepancia en la disciplina parental, siendo la madre quien intentaba fijar límites, los 
que no eran respaldados por el padre, cuando no abiertamente criticados 0 desautorizados. En algún momento de la segunda sesión, los niños $-y$ en especial luego que el paciente índice manifestó su rechazo y resentimiento por las peleas entre sus padres- adhirieron a esta declaración. Cuando este tema se puso en evidencia, Oscar expresó el lemor de que sus padres se volvieran a separar. Con posrerioridad a esta sesion se formuló la hipótesis de que la función del síntoma (o mejor dicho de los síntomas de Oscar) cumplía una función homeostática para mantener la integridad del sistema: el hijo se ofrece como "el problema" familiar en un intento de distraer la atención del problema conyugal y centrar la atención de ambos padres en sus problemas conductuales y evitar así que sus padres se separen. A su vez los padres se coluden consciente o subconscientemente también para congelar por un tiempo el problema conyugal y etiquetar cl problema familiar como "los problemas de conducta que Oscar les provoca".

Asi entonces en una sesión posterior se les comunicó al grupo familiar, previo a sostener una sesión sólo con ambos padres, que đe ahí en adelante se iba a trabajar solamente con ellos para ayudarles a resolver sus problemas (de tal manera de que los hijos pudieran estar más tranquilos y no necesitar ninguno de ellos salvar a la familia).

De ahí en adelante se llevó a efecto una terapia de pareja con resultados favorables, lo que redundó en una percepción de los hijos y de los padres que mejoró significativamente la dinámica y los "problemas conductuales" del paciente identificado.

\section{Comentario}

La terapia familiar sistémica comenzó a desarrollarse en nuestro país en la decada del año 1980. Con esta exposición se ha pretendido familiarizar al médico no especialista con este enfoque teórico y terapéutico e invitarlo a aplicar en su práctica profesional algunos de sus postulados básicos, sin que necesariamente tenga un entrenamiento formal. Lo anterior resulta particularmente necesario, dado el espectacular avance de la medicina que, junto con sus logros, ha generado múltiples subespecialidades que tienden progresivamente a la fragmentación del ser humano y a mediatizar la relación médicopaciente al extremo de hacer el encuentro personal cada yez más exiguo. El desafío mayor parecería entonces ser, hoy día, la recuperación del ser humano en su contexto biologico, psicológico y social. Esto supone -permanentementeconsiderar que el individuo influye en su contexto y es influido por este en secuencias repetidas de interacción.

Michael Balint, basado en un estudio realizado en Inglaterra, decía: "Aproximadamente un tercio de los casos de niños traídos al consultorio por sus padres, son estos los que necesitan tratamiento, en otro tercio tanto los hijos como los padres necesitan tratamiento y sólo en el tercio restante, el niño es el único enfermo".

A título de ejemplo, sería muy necesario ampliar este lente diagnóstico sistémico en nin̄os o adultos que tienden a enfermarse 0 a accidentarse reiteradamente (accident proneness) o a sufrir enfermedades de curso tórpido o atípico. La observación empírica sugiere que algunas personas enfrentadas a ciertos problemas o conflictos usan consciente o subconscientemente el recurso de enfermarse. Entre las situaciones más conocidas que tienden a generar dicha inclinación cabe destacar las necesidades afectivas insatisfechas; el sentimiento de incapacidad para enfrentar determinados desafíos (escolares, laborales u otros) que superan las capacidades que el individuo tiene o cree tener; el propósito de desviar la atención de terceros sobre otros conflictos (como el niño del primer caso, que temía la separación de sus padres); la agresividad reprimida; el estrés crónico; la falta de comunicación franca, directa y explícita; los estados depresivos larvados.

La interpretación más simple de base biológica podría ser, para algunos de estos casos, alguna "disminución de los mecanismos de defensa". El hecho que el laboratorio demuestre una deficiencia del sistema inmunológico no debiera excluir las variables psicosociales, si se reconoce la interacción dinámica que existe entre la biología y las emociones.

En una anamnesis orientada con este enfoque es importante averiguar si la familia es nuclear, extendida, uniparental (28\% en Chile), simultánea (mixta o reconstituida). En las extendidas indagar si el poder en la relación jerárquica reside en alguno de los padres o abuelos. Estos últimos pueden autorizar o boicotear en ocasiones 
la intervención médica. En las monoparentales conviene saber si el padre o madre es separado, soltero, viudo y qué fuentes de apoyo tiene. Tambien debe establecerse la etapa del ciclo familiar: si es una familia de recién casados; lleg6 hace poco un primer hijo: por primera vez entró cl niño al jardín infantil o a la escuela: si se trata de un adolescente o si todos los hijos están en la etapa juvenil; si se trata del "nido vacío". De particular importancia es averiguar si la madre trabaja fuera del hogar y en qué medida dicha situación no está terìida de culpas o ambivalencia y el apoyo que ella recibe de su pareja o esposo. El clima prevaleciente en el hogar (tenso o relajado) y el grado de avenencia conyugal pueden ser también factores significativos, del mismo modo que los hijos más "apegados" a mamá o papá. la reacción de los otros hijos a la llegada de uno nuevo, en especial del que hasta entonces era el menor. Las creencias religiosas, e] origen étnico, el nivel socioeconómico y cultural pucden resultar a veces igualmente relevantes.

\section{Referencias}

1. Montenegro $H$ : Terapia familiar sistérnica. En: Montenegro H, Guajardo $\mathrm{H}$. (Eds) Psiquiatria del Niño y del Adolescente. Editorial Salvador. Santiago, Chile, 1994.

2. Johorisen $O$ : Introducción a la Teoria General de Sistemas, Santiago. Facultad de Ciencias Económicas y Administrativas. Departamento de Administración. Universidad de Chile, 1975.

3. Garman $A$ and Kniskem DP: Handbook of Family Therapy. Brunner Mazel, Inc. New York, 1981.

4. Jackson $D$ : Interacción familiar, homeostasis familiar y psicoserapia familiar conjunca. En: Interaccion familiar. Buenos Aires. Tiempo Contemporáneo. 1971.

5. Haley J: Revisión del campo de la terapia familar. En: Tratomiebto de la familia. Barcelona Toray S.A. 1974.

6. Surir V: Psicorerapia familiar conjunta. Mexico. La Prensa Mexicana, 1982.

7. Watzlawick $P$, Beavin $J H$, Jacksun $D$ : Teoría de la comunicación humana. Barcélona. Bithlioteca Psicológica 100. Editorial Harder, 1981.

8. Miatichin S: Familias y terapia familiar, Barcelona. Gedisa, 1977.

9. Minuchin $S$ : Family therapy technique. Harvard University Press. USA, 1981. 